\title{
16S-23S rRNA gene internal transcribed spacer sequences for analysis of the phylogenetic relationships among species of the genus Porphyromonas
}

\author{
Georg Conrads, ${ }^{1,2}$ Diane M. Citron, ${ }^{1}$ Kerin L. Tyrrell, ${ }^{1}$ Hans-Peter Horz ${ }^{2}$ \\ and Ellie J. C. Goldstein ${ }^{1}$ \\ ${ }^{1}$ R. M. Alden Research Laboratory, Santa Monica, CA 90404, USA \\ ${ }^{2}$ Lehr- und Forschungsgebiet Orale Mikrobiologie und Immunologie der Klinik für \\ Zahnerhaltung, Parodontologie und Präventive Zahnerhaltung und Institut für Medizinische \\ Mikrobiologie, Universitätsklinikum (RWTH), D-52057 Aachen, Germany
}

Correspondence Georg Conrads gconrads@ukaachen.de

\section{INTRODUCTION}

The genus Porphyromonas currently includes 13 recognized species of asaccharolytic, obligately anaerobic, nonspore-forming, Gram-negative, non-motile, pleomorphic bacilli. Of human origin are three catalase-negative species: Porphyromonas asaccharolytica, Porphyromonas endodontalis and Porphyromonas gingivalis. Most of the known species are, however, of animal origin, including the catalasepositive Porphyromonas canoris, Porphyromonas cangingivalis,

Published online ahead of print on 24 September 2004 as DOI 10.1099/ijs.0.63234-0.

Abbreviations: ITS, internal transcribed spacer; PELO, 'Porphyromonas endodontalis-like organism'.

The GenBank/EMBL/DDBJ accession numbers for the ITS and 16S rRNA gene sequences discussed in this study can be found in Figs 1 and 2.

A similarity matrix table (.xls), an alignment file (.aln) and a multi sequence file (.msf) of DNA-DNA hybridization data for all sequences discussed in this article are available as supplementary material in IJSEM Online. A figure showing representative gel-electrophoretic patterns of Porphyromonas species is also available.
Porphyromonas cansulci, Porphyromonas circumdentaria, Porphyromonas gingivicanis and Porphyromonas macacae (which includes Porphyromonas salivosa strains), and the catalase-negative Porphyromonas levii and Porphyromonas crevioricanis (Jousimies-Somer \& Summanen, 2002). It has also been shown that Oribaculum catoniae, although saccharolytic, is phylogenetically a member of the genus Porphyromonas; thus, it has been reclassified as Porphyromonas catoniae (Willems \& Collins, 1995). Finally, animal strains of $P$. gingivalis that were catalase-positive were reclassified as Porphyromonas gulae; however, the difference between Porphyromonas gingivalis and Porphyromonas gulae appeared questionable by analysis of $16 \mathrm{~S}$ rRNA gene sequences (Fournier et al., 2001).

There are two additional candidates for new species classification, both of which are from humans: PLLOs ('Porphyromonas levii-like organisms'; Jousimies-Somer, 1995, 1997; Jousimies-Somer et al., 1995) and PELOs ('Porphyromonas endodontalis-like organisms', isolated from extra-oral sites, whereas Porphyromonas endodontalis is almost exclusively isolated from endodontic infections; Jousimies-Somer, 1997; Jousimies-Somer \& Summanen, 2002; Vaisanen 
et al., 1997). It should also be mentioned that there is a $16 \mathrm{~S}$ rRNA gene sequence for 'Porphyromonas canis' strain JCM 10100 in GenBank/EMBL/DDBJ (accession no. AB034799), but this name has never been formally proposed or validated. Within the proposed order 'Bacteroidales', Tannerella forsythensis (a species related to Bacteroides distasonis and Bacteroides merdae) is grouped within the proposed family 'Porphyromonadaceae', so that all three might be close relatives of Porphyromonas species (Sakamoto et al., 2002).

Sequence polymorphisms and length variations found in the 16S-23S rRNA gene internal transcribed spacer (ITS) are increasingly being used as tools for the differentiation of bacterial species and subspecies (Guasp et al., 2000; Motoyama \& Ogata, 2000; Conrads et al., 2002). This is because the higher number of variable sites typical for the ITS sequence (Soller et al., 2000) can overcome the apparent limitations of the resolution of $16 \mathrm{~S}$ rRNA-based phylogenies in some genera where amplification and sequencing of this region is easy, almost free of ambiguities and reproducible, as has been described for Fusobacterium (Conrads et al., 2002).

The current study was performed to generate ITS data for most of the type strains of the genus Porphyromonas and for T. forsythensis, B. distasonis and Prevotella melaninogenica (outgroup), and to compare a phylogenetic tree deduced from these data with one deduced from corresponding $16 \mathrm{~S}$ rRNA gene data. The ITS sequences were further used to clarify the phylogenetic relationship between Porphyromonas gingivalis and Porphyromonas gulae, as well as between atypical $\alpha$-fucosidase-negative isolates of Porphyromonas asaccharolytica (which are phenotypically indistinguishable from the PELO group; Vaisanen et al., 1997 ) and typical $\alpha$-fucosidase-positive Porphyromonas asaccharolytica.

\section{METHODS}

Bacterial strains, culture conditions and DNA extraction. The following bacterial strains were used: Porphyromonas asaccharolytica ATCC $25260^{\mathrm{T}}$; RMA (R. M. Alden Research Laboratory) 7115 (sacral wound); RMA 7120 (toe); RMA 7178 (endocervix); RMA 8631 (rectal abscess); RMA 9240 (peritoneal); RMA 9603 (abdominal); RMA 9674 (appendiceal fluid); RMA 10263 (peritoneal); RMA 10884, RMA 10898, RMA 10955, RMA 10966, RMA 10997, RMA 11049, RMA 11138 and RMA 11258 (all from pelvic fluid); RMA 11290 (vaginal cupule); RMA 11582 (endometrial pus); RMA 11690 (endometrium); RMA 11666 (endometrial pus); RMA 11805 (pelvic fluid); RMA 12959, RMA 12984 and RMA 13273 (all from diabetic foot infections); Porphyromonas cangingivalis NCTC $12856^{\mathrm{T}}$; Porphyromonas cansulci NCTC $12858^{\mathrm{T}}$; Porphyromonas circumdentaria NCTC $12469^{\mathrm{T}}$; Porphyromonas endodontalis ATCC $35406^{\mathrm{T}}$; Porphyromonas gingivalis ATCC $33277^{\mathrm{T}}$; RMA 3725 (oral, mandible); RMA 4165 (oral, maxilla); RMA 10371 (peritoneal/ abdominal fluid); Porphyromonas gulae ATCC $51700^{\mathrm{T}}$; Porphyromonas gingivicanis ATCC $55562^{\mathrm{T}}$; Porphyromonas levii ATCC $29147^{\mathrm{T}}$; Porphyromonas macacae ATCC 33141; Porphyromonas salivosa ATCC $49407^{\mathrm{T}}$; B. distasonis ATCC $8503^{\mathrm{T}}$; T. forsythensis ATCC $43037^{\mathrm{T}}$; and Prevotella melaninogenica ATCC $25845^{\mathrm{T}}$. The last three strains were used for contrast. All strains were cultivated at $37^{\circ} \mathrm{C}$ on Brucella agar (Anaerobe Systems, Morgan Hill, CA, USA) under anaerobic conditions using an anaerobic chamber. Genomic DNA was extracted using the DNeasy Tissue Kit (Qiagen).

PCR amplification and DNA sequence analysis. The $16 \mathrm{~S}$ primer SPFPorph (5'-GTA CAC ACC GCC CGT CAA GCC-3', corresponding to Escherichia coli positions 1390-1411) and the 23S primer SPRPorph (5'-TCG CAG CTT ATC ACG TCC TTC-3', corresponding to $E$. coli positions 62-42) were designed based on the complete genome of $P$. gingivalis W83 (GenBank/EMBL/DDBJ accession no. NC_002950); however, the respective regions among bacterial small-and large-subunit sequences (RDP) are relatively conserved. PCR was carried out using an Uno I (Biometra) thermocycler in a volume of $100 \mu \mathrm{l}$ containing $1 \times$ PCR buffer, $1.5 \mathrm{mM}$ $\mathrm{MgCl}_{2}, 2$ units Taq polymerase, $0.2 \mathrm{mM}$ each of dATP, dCTP, dGTP and dTTP (Roche Biochemicals), 10 pmol SPFPorph forward primer, $10 \mathrm{pmol}$ SPRPorph reverse primer and $100 \mathrm{ng}$ template DNA. Primer oligonucleotides were synthesized using a DNA synthesizer (OLIGO 1000; Beckman). The amplification was performed using the following temperature profile and 30 cycles: denaturation, $1 \mathrm{~min}$ at $94^{\circ} \mathrm{C}$; annealing, $1 \mathrm{~min}$ at $52^{\circ} \mathrm{C}$; elongation, $2.5 \mathrm{~min}$ at $72^{\circ} \mathrm{C}$. Amplification products (aliquots of $10 \mu \mathrm{l}$ ) were separated electrophoretically on a $2 \%$ macro agarose gel in $1 \times \mathrm{TPE}$ ( $80 \mathrm{mM}$ Tris/phosphate, $2 \mathrm{mM}$ EDTA, $\mathrm{pH} 7 \cdot 5)$ for a minimum of $18 \mathrm{~h}$ at $30 \mathrm{~V}$.

After purification using the Wizard DNA Clean-up system (Promega), the spacer DNA was directly sequenced in duplicate using a Big Dye-Deoxy terminator cycle sequencing kit (Applied Biosystems) and an automated capillary DNA sequencer (API Prism 310; Applied Biosystems). Sequences were assembled using the program Vector NTI Suite 9.0 (InforMax) and aligned using the program GeneDoc (Nicholas \& Nicholas, 1997) A phylogenetic tree was constructed by using the neighbour-joining method and the programs CLUSTAL W (Chenna et al., 2003) and TreeView (Page, 1996), using Prevotella melaninogenica as an outgroup. The robustness of tree topologies and the statistical significance levels of interior nodes were determined by performing bootstrap analyses (1000 iterations) using the ARB software package (Ludwig et al., 2004).

\section{RESULTS AND DISCUSSION}

Approximations of ITS lengths were obtained from agarose gels, as demonstrated in Fig. Aa (available as supplementary material in IJSEM Online). All Porphyromonas reference strains showed a single band between $970 \mathrm{bp}$ (Porphyromonas gingivalis ATCC $33277^{\mathrm{T}}$ ) and $710 \mathrm{bp}$ (Porphyromonas circumdentaria NCTC $12469^{\mathrm{T}}$ ). The four strains of Porphyromonas gingivalis analysed were almost identical by ITS amplicon length (970-960 bp) and sequence (97-99\% similarity). In contrast, among 24 clinical isolates of Porphyromonas asaccharolytica and the type strain ATCC $25260^{\mathrm{T}}$, the length of the ITS amplicons was more variable and ranged from $1044 \mathrm{bp}$ (Porphyromonas asaccharolytica RMA 10263, $\alpha$-fucosidase-negative strain) to $960 \mathrm{bp}$ (Porphyromonas asaccharolytica ATCC $25260^{\mathrm{T}}$, $\alpha$-fucosidase-positive strain) (Fig. Ab, available as supplementary material in IJSEM Online).

In general, it was not possible to differentiate Porphyromonas species by comparing ITS gel-electrophoretic profiles alone (for length of amplicons see supplementary material). Further discrimination without need of sequencing might be possible by ITS restriction digest with 
endonucleases, since we found considerable variation in restriction sites (e.g. AvaI, ApaLI, ClaI, EcoRI, HindIII and SmaI).

Sequencing the purified ITS amplicons of the Porphyromonas strains using SPFPorph and SPRPorph as primers led to nearly ambiguity-free sequence determination by comparing both runs and directions. Transfer RNA genes (type Ile and Ala) were found in all 'Porphyromonadaceae' ITS sequences but not in Prevotella melaninogenica, using the program tRNAscan-SE, version 1.21 (Lowe \& Eddy, 1997).
Phylogenetic tree reconstruction based on the ITS sequences (short version only in the case of Prevotella melaninogenica) is demonstrated in Fig. 1. As mentioned above, the different strains of Porphyromonas gingivalis matched on a 97-99\% level and the two Porphyromonas macacae ATCC strains (ATCC $49407^{\mathrm{T}}$ is the type strain of Porphyromonas salivosa, which has been reclassified) matched on a $94 \%$ level; however, Porphyromonas asaccharolytica was more heterogeneous $(80-99 \%$ range in similarity level). Even more interesting, this latter species, which phenotypically differed in $\alpha$-fucosidase activity, showed two main clusters. Intercluster similarity was only

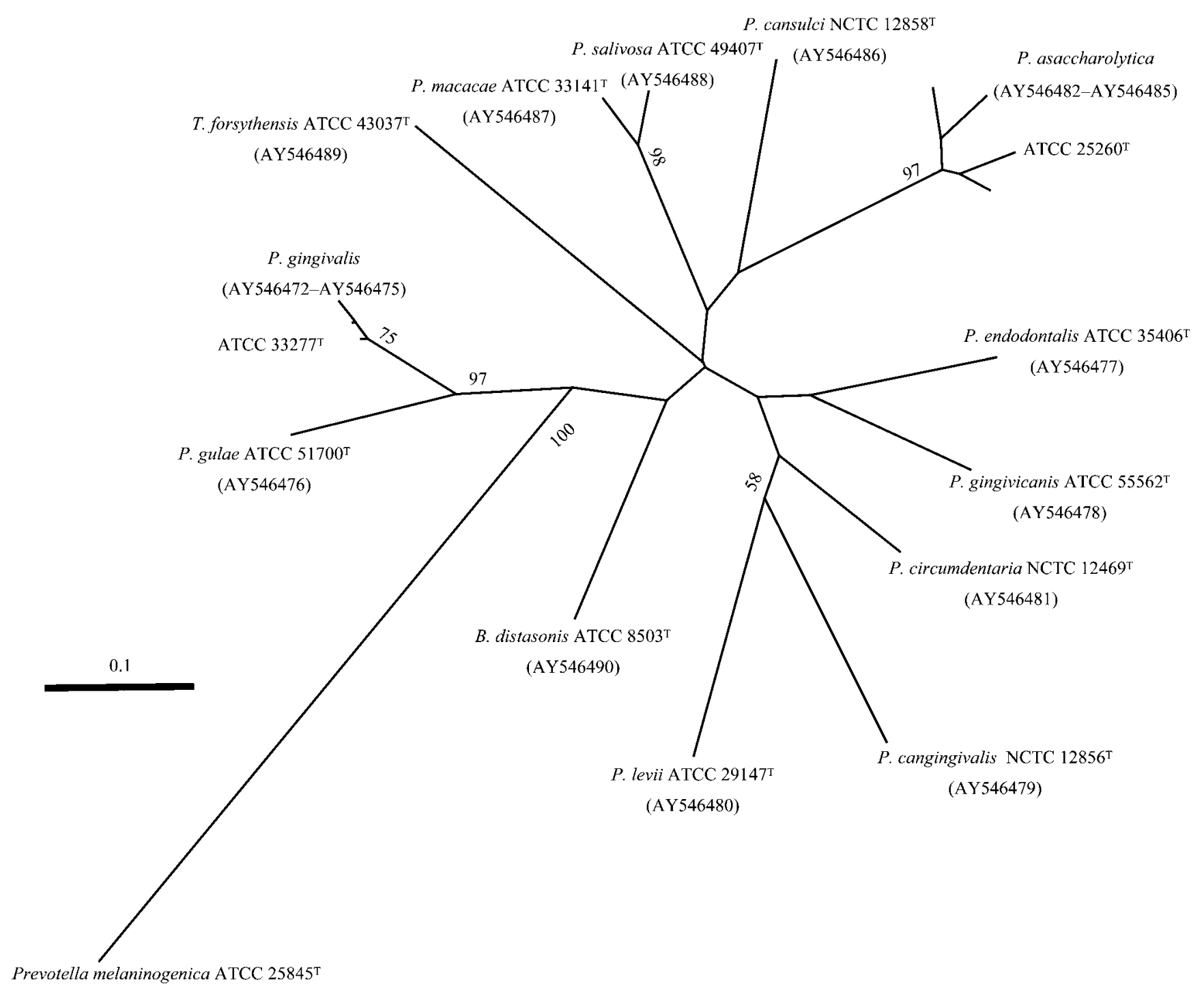

(AY546491)

Fig. 1. Phylogram (neighbour-joining method) showing the genetic relationships among Porphyromonas species based on

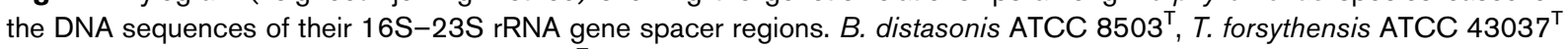
and Prevotella melaninogenica ATCC $25845^{\top}$ (outgroup, based on the short version of spacer) were included for contrast. The numbers at the nodes indicate the percentage recovery in 1000 bootstrap resamplings. Only bootstrap values above $50 \%$ are shown. Bar, $0 \cdot 1$ substitution per nucleotide. 
80-87\%, whereas intracluster similarity was $92-99 \%$ and thus more comparable with the phylogenetic relatedness of Porphyromonas gingivalis strains.

Based on the spacer sequences, four groups were distinguished. Porphyromonas gingivalis and Porphyromonas gulae represented an individual branch with $84-85 \%$ similarity to each other, justifying their separation into two different species. A second group was formed by Porphyromonas endodontalis/Porphyromonas gingivicanis and Porphyromonas circumdentaria $(75-81 \%)$ and a third group by Porphyromonas levii and Porphyromonas cangingivalis $(69 \%)$. The latter two groups were again related by ITS but not by $16 \mathrm{~S}$ information (Fig. 2). The fourth group was formed by Porphyromonas macacae, Porphyromonas asaccharolytica and Porphyromonas cansulci, which showed a match between 66 and $68 \%$.

T. forsythensis and B. distasonis showed $57 \%$ similarity to each other and were related by $43-70 \%$ to the Porphyromonas species and by $43-57 \%$ to Prevotella melaninogenica (43-57\%). With the exception of Porphyromonas cansulci, cluster analysis by ITS and $16 \mathrm{~S}$ information led to the same qualitative result. However, whereas the similarities calculated by ITS information were between $44 \%$ (Porphyromonas levii versus Porphyromonas gulae) and $85 \%$ (Porphyromonas gingivalis versus Porphyromonas gulae), similarities calculated by $16 \mathrm{~S}$ information were found to be much higher and between $83 \%$ (Porphyromonas asaccharolytica versus Porphyromonas catoniae) and $97 \%$

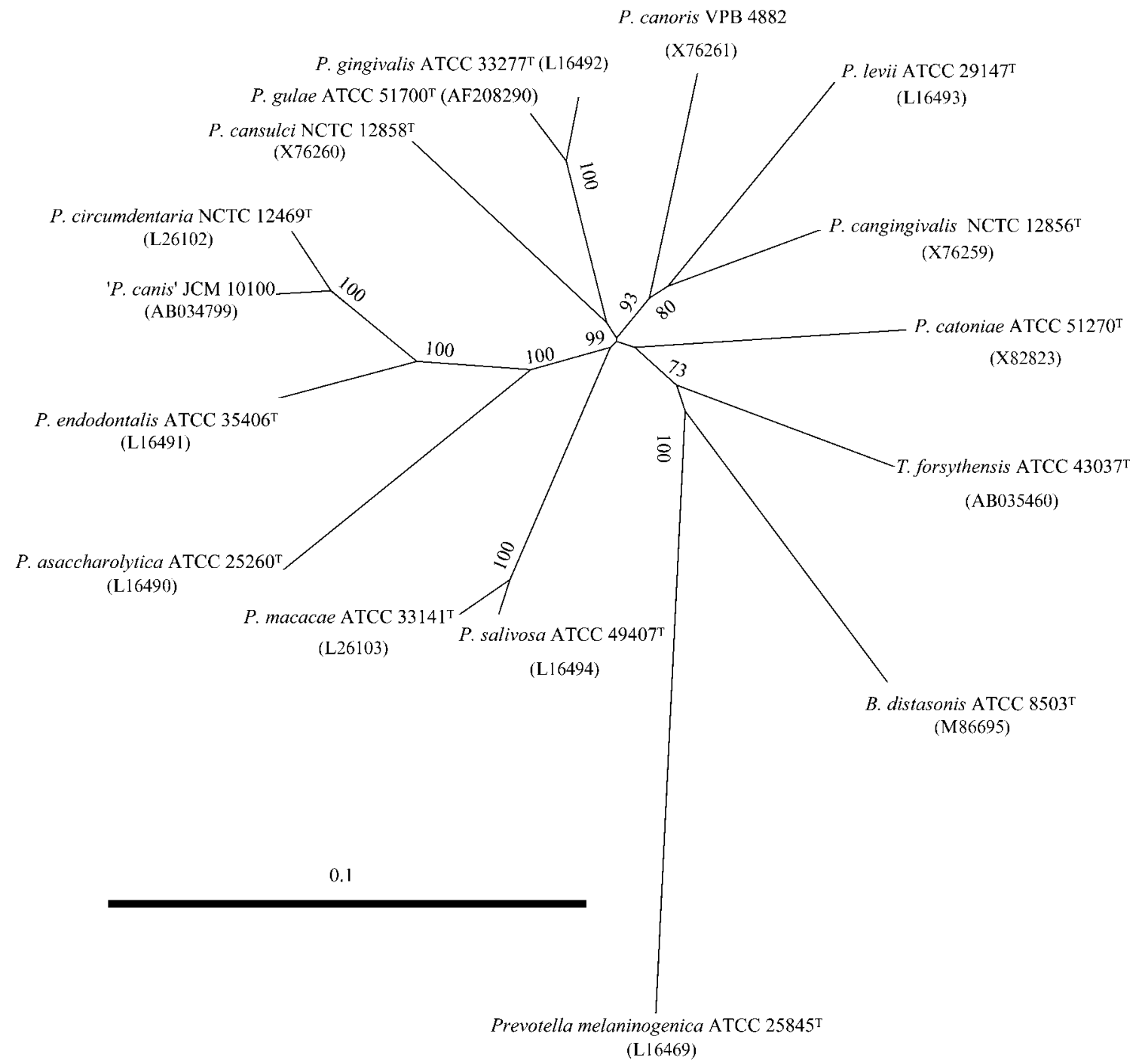

Fig. 2. Phylogram (neighbour-joining method) showing the genetic relationships among Porphyromonas species based on information from their $16 \mathrm{~S}$ rRNA gene sequences. B. distasonis ATCC $8503^{\top}, T$. forsythensis ATCC $43037^{\top}$ and Prevotella melaninogenica ATCC $25845^{\top}$ (outgroup) were used for contrast. The numbers at the nodes indicate the percentage recovery in 1000 bootstrap resamplings. Only bootstrap values above $50 \%$ are shown. Bar, $0 \cdot 1$ substitution per nucleotide. 
(Porphyromonas gingivalis versus Porphyromonas gulae), underlining the higher resolution potential by ITS sequence analyses. The higher resolution was further used to analyse the relationship between nine $\alpha$-fucosidase-positive and 16 $\alpha$-fucosidase-negative strains of Porphyromonas asaccharolytica and clearly showed that both groups diverged into individual phylogenetic branches (Fig. 3a). While the $\alpha$ fucosidase-negative strains phenotypically resemble the previously studied extra-oral isolates of PELOs, it is obvious that they are not genetically related to Porphyromonas endodontalis (Fig. 3b).

As we have previously reported for fusobacterial species, deducing bacterial phylogenetic relationships from 16S-23S rRNA gene ITS sequences seems to have several advantages over using 16S rRNA/DNA alone (Conrads et al., 2002), especially when comparing species that are closely related. The phylogenetic differences are not only expressed in the sequence information itself but also by the different lengths of amplicons and, in some cases, the formation of distinct band patterns by gel electrophoresis resulting from variations among the rrn operons in the same strain (Christensen et al., 2000; Iteman et al., 2000). However, in some members of the Proteobacteria and in low- $\mathrm{G}+\mathrm{C}$ Gram-positive genera such as Bacillus and Clostridium, six to ten rRNA operons exist, interfering with the reproducibility of ITS sequencing (Boyer et al., 2001). Furthermore, it was demonstrated by the same group that one strain might possess several ITS regions which are identical in length but differ by sequence. As our ITS sequences deduced from 38 Porphyromonas strains - were almost ambiguity-free, we conclude that the existence of multiple rRNA operons with significant difference in sequence in this genus is not very likely, which is in accordance with the findings of Nadkarni et al. (2002), who studied Porphyromonas endodontalis and other species using rRNA-operondirected real-time quantitative PCR.

PCR amplification of the ITS region using newly designed primers, and subsequent gel electrophoresis of 11 different Porphyromonas reference strains plus three clinical isolates of Porphyromonas gingivalis and 24 of Porphyromonas asaccharolytica, showed larger heterogeneity in length of amplicons in comparison to Fusobacterium species (Conrads et al., 2002). Furthermore, only one distinct amplification band was produced with Porphyromonas species as well as with the relatives $T$. forsythensis and $B$. distasonis, unlike Fusobacterium species (one to four bands; Conrads et al., 2002), Prevotella melaninogenica, Prevotella intermedia (two bands, data not shown) or many other genera analysed so far (Graham et al., 1997; Gurtler et al., 1999; Motoyama \& Ogata, 2000). However, judging by the electrophoretic pattern of Porphyromonas asaccharolytica strains, where a 50 bp shorter, 'shadow-like' second band can be seen (Fig. Ab, available as supplementary material in IJSEM Online), a second 16S-23S rRNA gene ITS sequence might exist. Within a species, the length of amplicons and the deduced sequence is relatively constant as we have shown
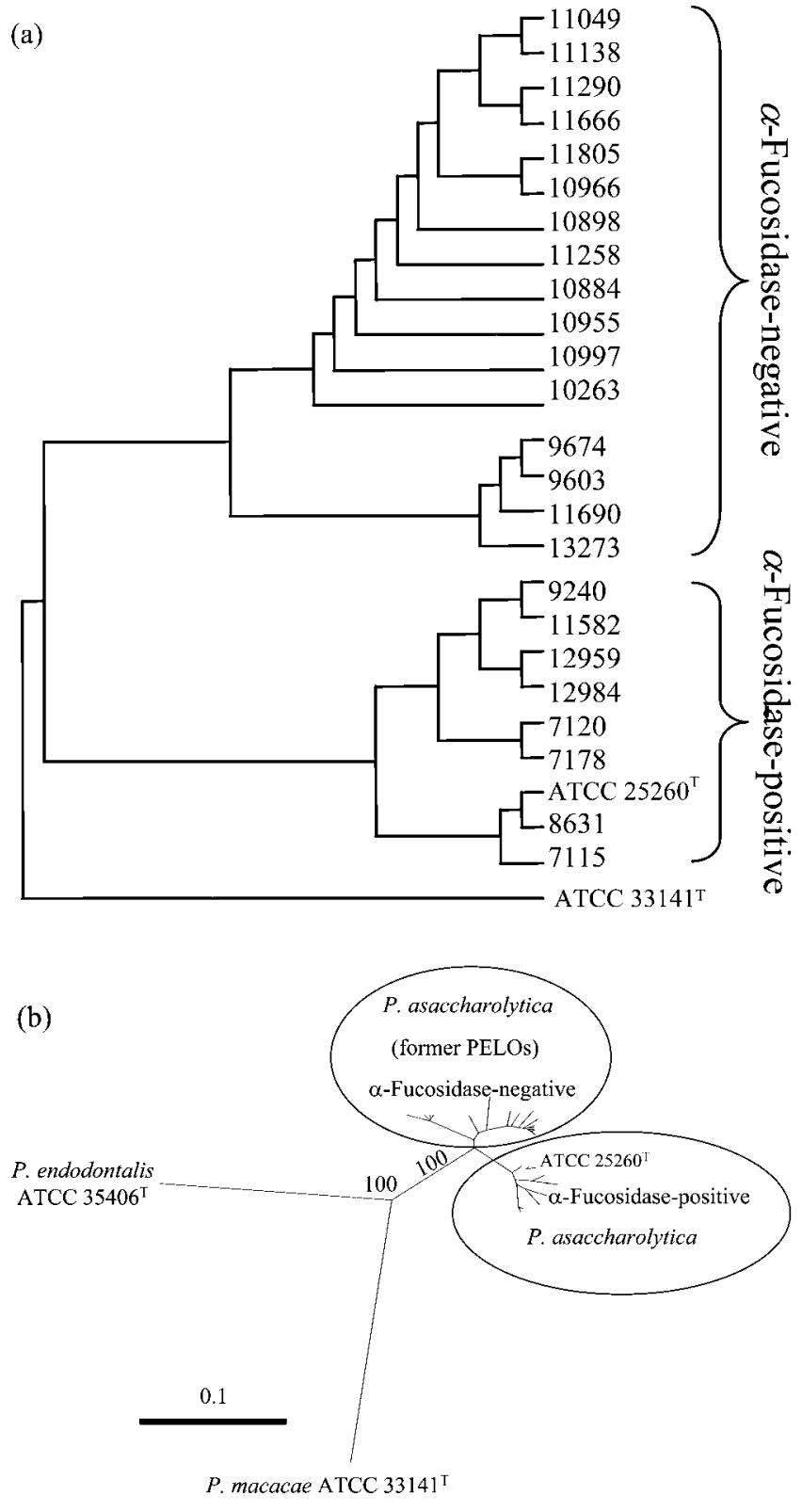

Fig. 3. (a) Cladogram showing the genetic relationships among 25 strains of Porphyromonas asaccharolytica including $16 \alpha$ fucosidase-negative strains, eight $\alpha$-fucosidase-positive strains and the type strain ATCC $25260^{\top}$. Porphyromonas macacae ATCC $33141^{\top}$ was used as outgroup. (b) The phylogram (neighbour-joining method) gives an overview about the genetic (ITS-based) distance of the $\alpha$-fucosidase-negative strains also known as PELOs and Porphyromonas endodontalis (ATCC $35406^{\top}$ ). They build a separate cluster in the Porphyromonas asaccharolytica branch of the genus Porphyromonas.

for Porphyromonas gingivalis (four strains), Porphyromonas macacae (two strains) and for fusobacterial species and subspecies. Thus, the heterogeneity found between the 25 Porphyromonas asaccharolytica strains could harbour an unrecognized species. 
The high resolution of ITS sequences led to a separation of Porphyromonas asaccharolytica strains into two clusters: one cluster was $\alpha$-fucosidase-positive, as is typical of the type strain, and the other was $\alpha$-fucosidase-negative and biochemically resembled the previously studied unusual extra-oral isolates of PELOs. PELOs have been isolated as part of the mixed flora from extra-oral infections in adults (for example, from appendicitis with or without peritonitis, infected sacral decubitus ulcer, infected mastoid bone and pilonidal abscess) and have also been found in faecal specimens from children (Finegold \& JousimiesSomer, 1997). However, the isolation of PELOs from extraoral sites is in contrast to the oral origin of Porphyromonas endodontalis but correlates with the Porphyromonas asaccharolytica-related cluster. By current biochemical methods, $\alpha$-fucosidase-negative Porphyromonas asaccharolytica strains cannot be differentiated from Porphyromonas endodontalis (Jousimies-Somer et al., 2002). Both are indole-positive, lack the typical glycosidase and peptidase enzymes, demonstrate fluorescence at $365 \mathrm{~nm}$ and do not ferment carbohydrates. In peptone/yeast/glucose (PYG) medium, acetic, propionic, butyric and isovaleric acids are produced as major metabolic products as well as isobutyric and succinic acids as minor products. The main cellular fatty acid is iso- $\mathrm{C}_{15: 0}$, followed by $3-\mathrm{OH}$ iso- $\mathrm{C}_{17: 0}, \mathrm{C}_{16: 0}$, iso- $\mathrm{C}_{13: 0}, 3-$ $\mathrm{OH}$ iso- $\mathrm{C}_{15: 0}, \mathrm{C}_{14: 0}$ and minor amounts of anteiso- $\mathrm{C}_{15: 0}$. This profile is not distinctively different from that of Porphyromonas asaccharolytica (Vaisanen et al., 1997) but it is different from that of Porphyromonas endodontalis, which produces neither $\mathrm{C}_{14: 0}$ nor $3-\mathrm{OH}$ iso- $\mathrm{C}_{15: 0}$, and less iso- $\mathrm{C}_{13: 0}$. Again, this supports our theory that PELOs are not related to $P$. endodontalis but are identical to $\alpha$ fucosidase-negative $P$. asaccharolytica strains.

The separation between Porphyromonas gingivalis and Porphyromonas gulae as distinct species was supported by our ITS data; thus, Porphyromonas gulae should not be referred to as the 'animal strain of Porphyromonas gingivalis' as it is genetically related but not identical to Porphyromonas gingivalis. Fournier et al. (2001), describing Porphyromonas gulae, pointed out the paradox that although this species could be distinguished from Porphyromonas gingivalis phenotypically and by DNA-DNA similarity, the differences between genes encoding 16S rRNA were small. They also concluded that the recent divergences of ancestral phyla (for example, after colonizing different mammalian hosts) could not be sufficiently discerned by $16 \mathrm{~S}$ information. Again, at least in some genera, ITS data give additional information and enhance phylogenetic resolution if discrepancies between DNA-DNA hybridization and $16 \mathrm{~S}$ sequencing results are observed.

In conclusion, the ITS region is being used increasingly as an important tool for classification and differentiation of bacterial species. Our study is the first to provide this sequence information for most of the Porphyromonas species and their relatives. The higher resolution of ITS sequences helped clarify some of the current problems in molecular taxonomy. In addition, we found that the name PELO should no longer be used for $\alpha$-fucosidase-negative strains of Porphyromonas asaccharolytica that resemble Porphyromonas endodontalis. Instead, the name PALO ('Porphyromonas asaccharolytica-like organism') would be more suitable. Moreover, because of the reduced ITS similarity $(80-85 \%)$ between the $\alpha$-fucosidase-positive Porphyromonas asaccharolytica ATCC $25260^{\mathrm{T}}$ and the $\alpha$ fucosidase-negative cluster, the proposal of a new species seems to be warranted (Finegold et al., 2004).

\section{ACKNOWLEDGEMENTS}

We thank Yumi Warren, Ilse Seyfarth, Helen T. Fernandez, Vreni Merriam, Alice E. Goldstein and Gisela Conrads for various forms of assistance.

\section{REFERENCES}

Boyer, S. L., Flechtner, V. R. \& Johansen, J. R. (2001). Is the 16S$23 \mathrm{~S}$ rRNA internal transcribed spacer region a good tool for use in molecular systematics and population genetics? A case study in cyanobacteria. Mol Biol Evol 18, 1057-1069.

Chenna, R., Sugawara, H., Koike, T., Lopez, R., Gibson, T. J., Higgins, D. G. \& Thompson, J. D. (2003). Multiple sequence alignment with the Clustal series of programs. Nucleic Acids Res 31, 3497-3500.

Christensen, H., Moller, P. L., Vogensen, F. K. \& Olsen, J. E. (2000). Sequence variation of the $16 \mathrm{~S}$ to $23 \mathrm{~S}$ rRNA spacer region in Salmonella enterica. Res Microbiol 151, 37-42.

Conrads, G., Claros, M. C., Citron, D. M., Tyrrell, K. L., Merriam, V. \& Goldstein, E. J. C. (2002). 16S-23S rDNA internal transcribed spacer sequences for analysis of the phylogenetic relationships among species of the genus Fusobacterium. Int J Syst Evol Microbiol 52, 493-499.

Finegold, S. M. \& Jousimies-Somer, H. (1997). Recently described clinically important anaerobic bacteria: medical aspects. Clin Infect Dis 25, S88-S93.

Finegold, S. M., Vaisanen, M. L., Rautio, M., Eerola, E., Summanen, P., Molitoris, D., Song, Y., Liu, C. \& JousimiesSomer, H. (2004). Porphyromonas uenonis sp. nov., a pathogen for humans distinct from $P$. asaccharolytica and P. endodontalis. J Clin Microbiol 42, 5298-5301.

Fournier, D., Mouton, C., Lapierre, P., Kato, T., Okuda, K. \& Menard, C. (2001). Porphyromonas gulae sp. nov., an anaerobic, Gram-negative coccobacillus from the gingival sulcus of various animal hosts. Int J Syst Evol Microbiol 51, 1179-1189.

Graham, T. A., Golsteyn-Thomas, E. J., Thomas, J. E. \& Gannon, V. P. (1997). Inter- and intraspecies comparison of the $16 \mathrm{~S}-23 \mathrm{~S}$ rRNA operon intergenic spacer regions of six Listeria spp. Int J Syst Bacteriol 47, 863-869.

Guasp, C., Moore, E. R. B., Lalucat, J. \& Bennasar, A. (2000). Utility of internally transcribed $16 \mathrm{~S}-23 \mathrm{~S}$ rDNA spacer regions for the definition of Pseudomonas stutzeri genomovars and other Pseudomonas species. Int J Syst Evol Microbiol 50, 1629-1639.

Gurtler, V., Rao, Y., Pearson, S. R., Bates, S. M. \& Mayall, B. C. (1999). DNA sequence heterogeneity in the three copies of the long 16S-23S rDNA spacer of Enterococcus faecalis isolates. Microbiology 145, 1785-1796.

Iteman, I., Rippka, R., Tandeau De Marsac, N. \& Herdman, M. (2000). Comparison of conserved structural and regulatory domains 
within divergent 16S rRNA-23S rRNA spacer sequences of cyanobacteria. Microbiology 146, 1275-1286.

Jousimies-Somer, H. R. (1995). Update on the taxonomy and the clinical and laboratory characteristics of pigmented anaerobic gramnegative rods. Clin Infect Dis 20, S187-S191.

Jousimies-Somer, H. (1997). Recently described clinically important anaerobic bacteria: taxonomic aspects and update. Clin Infect Dis 25, S78-S87.

Jousimies-Somer, H. \& Summanen, P. (2002). Recent taxonomic changes and terminology update of clinically significant anaerobic gram-negative bacteria (excluding spirochetes). Clin Infect Dis 35, S17-S21.

Jousimies-Somer, H. R., Summanen, P. \& Finegold, S. M. (1995). Bacteroides levii-like organisms isolated from clinical specimens. Clin Infect Dis 20, S208-S209.

Jousimies-Somer, H., Summanen, P., Citron, D. M., Baron, E. J., Wexler, H. M. \& Finegold, S. M. (editors) (2002). Wadsworth-KTL Anaerobic Bacteriology Manual, 6th edn. Belmont: Star Publishing.

Lowe, T. M. \& Eddy, S. R. (1997). tRNAscan-SE: a program for improved detection of transfer RNA genes in genomic sequence. Nucleic Acids Res 25, 955-964.

Ludwig, W., Strunk, O., Westram, R. \& 29 other authors (2004). ARB: a software environment for sequence data. Nucleic Acids Res 32, 1363-1371.

Motoyama, Y. \& Ogata, T. (2000). 16S-23S rDNA spacer of Pectinatus, Selenomonas and Zymophilus reveal new phylogenetic relationships between these genera. Int J Syst Evol Microbiol 50, 883-886.

Nadkarni, M. A., Martin, F. E., Jacques, N. A. \& Hunter, N. (2002). Determination of bacterial load by real-time PCR using a broad-range (universal) probe and primers set. Microbiology 148, 257-266.

Nicholas, K. B. \& Nicholas, H. B. J. (1997). GeneDoc: a tool for editing and annotation multiple sequence alignments. Distributed by the authors (http://www.psc.edu/biomed/genedoc).

Page, R. D. (1996). TreeView: an application to display phylogenetic trees on personal computers. Comput Appl Biosci 12, 357-358.

Sakamoto, M., Suzuki, M., Umeda, M., Ishikawa, L. \& Benno, Y. (2002). Reclassification of Bacteroides forsythus (Tanner et al. 1986) as Tannerella forsythensis corrig., gen. nov., comb. nov. Int J Syst Evol Microbiol 52, 841-849.

Soller, R., Hirsch, P., Blohm, D. \& Labrenz, M. (2000). Differentiation of newly described Antarctic bacterial isolates related to Roseobacter species based on 16S-23S rDNA internal transcribed spacer sequences. Int J Syst Evol Microbiol 50, 909-915.

Vaisanen, M. L., Kiviranta, M., Summanen, P., Finegold, S. M. \& Jousimies-Somer, H. R. (1997). Porphyromonas endodontalis-like organisms from extraoral sources. Clin Infect Dis 25, S191-S193.

Willems, A. \& Collins, M. D. (1995). Reclassification of Oribaculum catoniae (Moore and Moore 1994) as Porphyromonas catoniae comb. nov. and emendation of the genus Porphyromonas. Int J Syst Bacteriol 45, 578-581. 